\title{
FIBROMIALGÍA, DESIGUALDAD SOCIAL Y GÉNERO. ESTUDIO CUALITATIVO SOBRE TRANSFORMACIÓN DEL MALESTAR CORPORAL EN MALESTAR PSICOSOCIAL
}

\section{FYBROMIALGYA, SOCIAL INEQUITIES AND GENDER. QUALITATIVE STUDY ABOUT THE TRANSFORMATION OF BODY DISCOMFORT INTO PSYCHOSOCIAL DISCOMFORT}

TÍTULO CORTO: FIBROMIALGÍA, DESIGUALDAD SOCIAL Y GÉNERO

Margot Pujal I-Llombart $^{1}$, Enrico Mora-Malo ${ }^{2}$, Nicolás Schöngut-Grollmus ${ }^{3}$

Recibido en junio 16 de 2016

Aceptado en agosto 08 de 2016

\section{RESUMEN}

En los últimos años han aparecido problemáticas de salud nuevas y renovadas, cuya característica transversal es que no se les encuentra una causa orgánica en términos biopsicomédicos. Este artículo aborda algunas de éstas problemáticas asociadas al dolor crónico con diagnóstico de fibromialgia. De esta manera, el objetivo es analizar la forma en que personas con diagnóstico de fibromialgia y que participan en organizaciones de pacientes, transforman el malestar corporal en malestar psicosocial respecto a su enfermedad. Esto se ha hecho mediante el uso de métodos cualitativos, específicamente con grupos focales y análisis de contenido. Los principales resultados arrojan el mejoramiento de la calidad de vida en quienes logran hacer este tránsito, evidenciando al mismo tiempo la necesidad de incorporar las dimensiones de género, desigualdad social y relaciones de poder que cruzan el acceso a la salud pública en este nuevo conjunto de problemáticas sanitarias.

Palabras clave: Equidad en salud; dolor crónico; género y salud; fibromialgia

\section{ABSTRACT}

The research identifies the effects of sociodemographic variables and quality of life in the academic performance of 465 students of the Faculty of Health Sciences of the University of Magdalena. The methodology used for data collection consisted of the application of two instruments to students of the Faculty of Health Sciences of the University of Magdalena, the instrument of Martha Artunduaga on variables that influence the achievement of the University and the instrument SF 36 was the quality of life in health. We conclude that there is no relationship between quality of life, sociodemographic factors and academic performance in the subjects of this sample. While

1. Profesora Titular, Departamento de Psicología Social. Universitat Autònoma de Barcelona, España. Correo: margot.pujal@uab.cat. http://orcid.org/0000-0002-4823-2949. 2. Profesor Agregado, Departamento de Sociología. Universitat Autònoma de Barcelona, España. Correo: Enrico.Mora@uab.cat. http://orcid.org/0000-0002-9118-0398. 3. Profesor Asociado, Facultad de Psicología. Universidad Gabriela Mistral. Santiago, Chile. Correo: nicolas.schongut@ugm.cl. http://orcid.org/0000-0003-0066-7190. 
a significant and inverse relationship between age and academic performance as well as the social status and academic performance was observed, showing that the lower the social stratum have better academic performance among younger and better academic performance, are relates this phenomenon by the fact that students are faced with strength and persistence of the difficulties perceiving education as an opportunity to overcome poverty as the core of the theory of social mobility.

Keywords: Traditional Culture; Quality of life; performance; university; Social mobility

\section{INTRODUCCIÓN}

$E_{\mathrm{d}}^{\mathrm{n}}$ n las sociedades capitalistas y tecnológicamente desarrolladas contemporáneas están emergiendo un conjunto de problemas de salud nuevos y renovados, cuya característica transversal es que no se les encuentra una causa orgánica en términos biopsicomédicos. Dichos malestares corresponden a un $30 \%$ de la población ${ }^{1,2}$ e implican un aumento de la demanda de atención en salud (entre un $25 \%$ y $50 \%$ ) puesto que tienen un impacto limitante en la vida personal, social, familiar y laboral de las personas afectadas y, por tanto, también un impacto económico considerable en la sanidad pública. Sin embargo, aunque no se les encuentre causa orgánica desde paradigmas biopsicomédicos, ello no es un impedimento para que no se recurra frecuentemente a un intenso tratamiento farmacológico y/o psicofarmacológico en su tratamiento, que a menudo agrava y cronifica el problema ${ }^{3}$. Además, se da una clara morbilidad diferencial entre sexos, en este tipo de malestares, afectando a un $80 \%$ de mujeres y a un $20 \%$ de varones ${ }^{4}$. Por otra parte dichos malestares se asocian al cuerpo, usualmente en la forma de fibromialgia (FM), problemas osteoarticulares y músculo-esqueléticos, o fatiga crónica, entre los más relevantes, como también a la psique, encarnado como depresión crónica, fobias, las crecientes sociopatías, ansiedad, etc ${ }^{5}$.

Se trata de malestares que son más frecuentes en las sociedades globalizadas y neoliberales actuales, debido a un aumento exponencial en las últimas décadas del sufrimiento evitable, que es fruto de condiciones sociales, culturales y de poder adversas a la vida, como diferentes violencias simbólicas y estructurales, que afectan sobre todo a los grupos más vulnerables en los que confluyen e interactúan diferentes ejes de poder y desigualdad ${ }^{6-8}$. Cabe mencionar que las relaciones entre salud y desigualdades de género han sido documentadas y trabajadas desde hace décadas, siendo esta una perspectiva que permite no perder de vista las relaciones de poder que atraviesan estas problemáticas 9.

En el caso del dolor cronificado y el diagnóstico de fibromialgia, estos malestares, por sus características específicas, desbordan el paradigma biopsicomédico en el sentido de que al no encontrárseles una causa orgánica mediante las teorías y herramientas biomédicas o psicológicas al uso, se generan dudas sobre su existencia real y se convierten en malestares controvertidos en la arena social, sanitaria y jurídica ${ }^{10}$. El efecto de dicha controversia produce la mayoría de las veces un grado importante de indefensión y estigma en las personas afectadas, ya sea por el reconocimiento incierto de su dolencia o por la falta de tratamiento adecuado, y un malestar o experiencia de burnout en los profesionales que los atienden, cuando no conflictos profesionales entre éstos o saturación de los servicios de salud ${ }^{11}$.

Además, la disciplina psicológica, enfocada al cuidado de la salud en otro sentido, tampoco aborda el problema desde una perspectiva biopsicosocial y concepción integral de la salud. Esto se debe por una parte a que la psicología tiene una presencia reducida y muchas veces subsidiaria de la biomedicina, mientras que por otra a un reconocimiento escaso en la sanidad pública cuando es pensada desde el modelo biomédico. Por la otra parte, el paradigma biopsicomédico trabaja hegemónicamente en el ámbito público desde paradigmas, tiene como efecto una desestimación, en gran parte, del impacto en la salud de los factores culturales y psicosociales.

En el caso español, sin embargo, cada vez es más frecuente que esta indefensión y estigma se gestionen en algún grado por parte de las personas afectadas, a través de la creación propia de organizaciones de pacientes que son heterogéneas y ajenas a la organización de la 
sanidad pública. Estas formas de organización en torno al dolor cronificado con diagnóstico de fibromialgia se han ido replicando en otros países durante los últimos años, por ejemplo en Chile (Fibromialgía en Acción) y en Argentina (Asociación Fibroamérica). Son organizaciones comparten un objetivo transversal orientado a darse apoyo entre las pacientes y a conseguir reconocimiento y una legitimación, tanto privada como pública, de las experiencias de malestar.

Este artículo se centra en uno de estos malestares emergentes el dolor crónico sin causa orgánica, denominado Fibromialgia (FM), en el marco de la sanidad pública española. Este malestar crece de forma exponencial en el estado español y demás países occidentalizados tal y como se ha visto en una investigación precedente. El objetivo del artículo es presentar un análisis crítico a partir de un enfoque de género en salud ${ }^{11}$, dado que la FM se diagnostica mayoritariamente a mujeres, con un ratio mujer/hombre de $10 / 1^{12}$. En este sentido, el sistema sexo/género ${ }^{13}$ se considera un factor cultural y psicosocial central y se entiende como dispositivo de poder ${ }^{14}$. En esta línea el sujeto que enferma es conceptualizado como un sistema biopsicosocial dinámico: el sistema "sujeto/ sexo/género"2.

Desde esta perspectiva, la investigación se cuestiona por el género como un determinante psicosocial de la FM y se plantea la siguiente pregunta de investigación: ¿Cómo las organizaciones de pacientes de FM como nuevo contexto de apoyo y reconocimiento intersubjetivo posibilitan transformar la experiencia de dolor corporal cronificado en una experiencia psicosocial, relacionada con la transformación de la identidad de género? Dicho esto, el objetivo general de este artículo es analizar la forma en que personas con diagnóstico de fibromialgia y que participan en organizaciones de pacientes, transforman el malestar corporal en malestar psicosocial respecto a su enfermedad.

Se ha podido evidenciar la incorporación del género como variable en el estudio del dolor crónico y diagnósticos asociados durante los últimos años (por ejemplo FM o síndrome de fatiga crónica), en el contexto iberoamericano.

72 Estos confirman la mayor prevalencia de cuadros de este tipo en mujeres ${ }^{15}$, así como también la relación de este tipo de cuadros con otros diagnósticos, tales como ansiedad y/o depresión ${ }^{16}$. Sin embargo, muchos de estos estudios encuentran un límite en el uso de la variable género descriptivamente y no analíticamente. Por lo mismo, no incorporan elementos de la perspectiva de género tales como su capacidad de organización de la vida social y las relaciones de poder ${ }^{14}$ entre otros. Por el mismo motivo, si bien los criterios establecidos en manuales diagnósticos pueden ser de utilidad, son al mismo tiempo insuficientes ya que diagnósticos como FM son diagnósticos de exclusión y que no pueden ser confirmados con pruebas objetivas $^{17,18}$.

La organización sexista de la vida social genera unas fuentes específicas de vulnerabilidad y fragilidad diferenciadas según el sexo y el género ${ }^{19}$. Tres son las características fundamentales de una organización sexista occidental: La división sexual del trabajo, la construcción imaginaria, simbólica y material de las subjetividades de género y las relaciones sociales mediatizadas por esta categoría. Sin embargo, hay variaciones en las combinaciones de estas características que han dado lugar a distintos modelos sociales de género en el estado español y en otros territorios. En la actualidad, conviven cuatro modelos sociales de género que pueden interactuar entre ellos $^{2}$. Se trata de los modelos tradicional, en transición, contemporáneo e igualitario, señalados en el análisis de resultados.

\section{MATERIALES Y MÉTODOS}

El presente es un estudio cualitativo, en tanto este tipo de aproximación a la investigación permite una comprensión del mundo, del fenómeno y del problema de investigación desde la perspectiva de quienes participan y viven cotidianamente la FM en este caso, experimentando sus efectos y sus malestares día a día ${ }^{20}$. En cualquier lugar que exista un grupo de personas con una vida en común, se desarrolla una forma de vida en sí misma que cobra sentido y significado ${ }^{21}$. Dado que el interés en este artículo es acercarse y comprender los tránsitos subjetivos de mujeres que padecen dolor cronificado con diagnóstico de FM, migrando de un malestar corporal a un malestar psicosocial, se quiere comprender sus lugares iniciales y finales de las trayectorias, y también sus procesos, caminos, y complejidades, siendo así lo cualitativo una herramienta privilegiada para esta forma de trabajo ${ }^{22}$.

Para el levantamiento de información se ha escogido la técnica de los grupos focales, con participantes de organizaciones de pacientes con diagnóstico clínico de FM. En los grupos focales se pueden distinguir los grupos focales clínicos ${ }^{23,24}$ que se han utilizado en esta investigación, en tanto proveen a las personas investigadoras de insights respecto a los aspectos psicológicos de los sujetos participantes. 
Respecto a la constitución de los grupos focales, la muestra de personas entrevistadas eran personas diagnosticadas clínicamente con FM de hace más de tres años, y también personas que participan desde hace al menos dos años en algún tipo organización de pacientes de FM, con un papel protagonista y activo en la vida pública y privada de las afectadas. Las participantes procedían de servicios públicos, predominantemente primarios y secundarios, pertenecientes a la comunidad autónoma de Cataluña, una de las más activas en esta problemática ${ }^{25}$. El trabajo de campo fue realizado entre marzo del 2010 y enero del 2012.

En este estudio se ha diseñado una muestra estratégica de grupos focales a partir de los distintos tipos de organización existentes y de forma proporcional a la presencia en el territorio. La muestra final se compone de un total de 10 grupos focales, conformados por 85 personas, de las que solo 5 son varones. Cada grupo estuvo compuesto por entre 7 y 10 personas. Los grupos focales quedaron constituidos de la siguiente manera:

Siete grupos focales con integrantes de ACAF (Asociación Catalana de Afectados por la Fibromialgia), la organización más numerosa en Cataluña. Los grupos se han construido según el criterio de equilibrar asociaciones de zonas urbanas grandes y de poblaciones pequeñas.

Dos grupos focales a partir de Grupos de Ayuda Mutua (GAM) de FM que son independientes entre ellos y también independientes de ACAF.

Un grupo focal a partir de un grupo psicoterapéutico de FM. Estos son poco frecuentes pero existen, contando con el acompañamiento voluntario de un médico y una enfermera pertenecientes a un servicio público.

Como estrategia de análisis se utiliza el análisis de contenido ${ }^{25-27}$ incorporando un enfoque de género en salud, y con la ayuda del programa ATLAS-ti 6.0.

La estrategia de análisis de contenido utilizada es de carácter cualitativo e interpretativo, en tanto así es posible tanto describir cómo interpretar las trayectorias transformativas del malestar corporal al malestar psicosocial en pacientes con diagnóstico de FM. Asimismo, se utilizó una estrategia de codificación inductiva, vale decir producir las categorías de análisis a partir de los relatos y respuesta que emergen en los grupos focales para la elaboración de categorías. La categorización inductiva favorece el desarrollo de procesos interpretativos por parte de las personas investigadoras, mediante la lectura y la revisión del material ${ }^{25}$.

\section{Declaración de Aspectos Éticos}

Antes de los Grupos Focales se explicaron los objetivos del estudio, y se obtuvo el consentimiento informado, garantizándose a los informantes la confidencialidad y anonimato en la recolección y análisis de la información. La participación en los grupos focales fue voluntaria. En todo momento el proyecto adhirió de forma total a la Declaración de Helsinki, evaluando permanentemente la relación costo-beneficio del estudio para las personas, y la protección de grupos vulnerables específicos.

\section{RESULTADOS}

\section{Dolor y diferencia sexual biológica}

Aunque la FM afecta mayoritariamente a mujeres, cuando las afectadas hablan, al inicio de los grupos focales, sobre las posibles causas del malestar, y a pesar de hablar respecto a ellas explícitamente con una declinación femenina, no se refieren a su experiencia vital y condición de mujeres como factor psicosocial. En su lugar, la mayoría de veces aluden a factores biológicos diversos (por ejemplo, causas genéticas, neuropsicológicas, virales, etc.) y a la diferencia sexual biológica.

"Yo siempre pienso que tiene que haber algo en el organismo nuestro que sea diferente al de ellos (...) yo pienso, dentro de mí, que es que en nosotras hay algo, un líquido o yo que sé, qué provoca esto" (GP).

En el relato, la emergencia del dolor en el cuerpo es construida como ajena al sujeto y a su posición subjetiva, escenificándose la escisión cuerpo-mente cartesiana, que es propia del paradigma biomédico y, por supuesto, también es ajena a lo social y cultural.

"Yo era feliz en mi vida hasta que me llego esta putada, le tenía rabia a mi cuerpo porque me dolía" (GP).

"Con el cáncer sabes que te curas (...) de la FM sabes que ni te curas y que te fastidia, te fastidia de por vida. (...) Te va matando poco a poco; te mata ilusiones; ;te cambia la vida total!" (GFA1).

\section{Identidad de género normativa y vulnerabili- zación del yo}

Cuando se pregunta explícitamente en los grupos focales sobre la experiencia, los ideales y las expectativas en relación al hecho de ser mujeres a través de la trayectoria

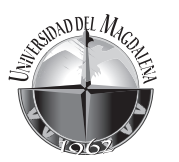


vital y los contextos de vida, sin establecer ninguna relación directa entre la FM y ésta, el resultado es la presencia de un relato muy repetido en el que destaca la conciencia de haber vivido en el pasado, en una posición subjetiva de seres para otros sin haber establecido límites, en contraposición a la posición de ser de $s^{28}$ :

"Nunca he sido niña porque cuando era pequeña (...) tenía que lavar la ropa de mis hermanos y mis primos, mi abuela me enseñaba a lavar, fregar; o sea, que yo recuerdo estar cuidando siempre, primos y hermanos" (GP).

"Primero era la madre, luego el marido y luego los hijos. Porque de alguna manera te imponen cosas. Entonces tú nunca has sido tú" (GFA4).

\section{Participación de modelos de género tradicional y en transición}

De los cuatro modelos sociales de género vigentes en las sociedades occidentales, hay dos que interpelan especialmente a las mujeres afectadas por la FM, el modelo tradicional y el modelo en transición. La diferencia fundamental entre ambos es la división sexual del trabajo. En el tradicional, esta división es general; en el modelo en transición, la división se mantiene sustantivamente en el espacio privado en relación a las tareas de cuidado y domésticas, pero se flexibiliza en relación al público en el sentido que las mujeres participan de trabajos remunerados. En este sentido, este modelo de transición genera contradicciones y ambivalencias en relación a las identidades de género y crea tensiones en las relaciones sociales.

En el caso de las mujeres diagnosticadas con FM, ellas han crecido introyectando el modelo tradicional. $\mathrm{Y}$, posteriormente, cuando ya han sido adultas, han incorporado el modelo en transición supuestamente más igualitario. Dicha superposición intensifica las contradicciones y conflictos vitales de ambos modelos.

"Y la comida, y la ropa, y cinco que éramos en mi casa, y la niña seis y lo hacía yo todo. No me ayudaban nada" (GP).

74 "Me dejé una cosa que para mí me encantaba y era mi carrera de música (...) pero lo dejé, ves, por ayudar demasiado a los demás, por entregarme demasiado a los demás" (GP).

El modelo en transición es en cambio la elección deseada voluntariamente, y es el denominador común de las familias de las personas entrevistadas, a lo largo de un periodo largo de su vida adulta. Este modelo acostumbra a ser el escenario de la emergencia del dolor y el diagnóstico de FM.

"Ibas de vacaciones y me iba con el teléfono, la responsabilidad continuaba, o sea... Yo en mi caso no he vivido para mí, he vivido para trabajar (...) pienso que mi trabajo era como una adicción y la familia era la continuación de la adicción" (GFA5).

"Hemos sido mujeres que hemos tenido que batallar con el hombre que no ayuda en casa, y hemos tenido que llevar una vida fuera de casa, una doble vida, sin la ayuda ni comprensión ni de la generación anterior ni de la nuestra" (GFA2).

\section{Vulnerabilidad psicosocial y dolor corporal}

La subjetivación de género en los contextos de desigualdad señalados, no puede sino ser fuente de vulnerabilidad, que en el caso de este trabajo se expresa a través de una vulnerabilidad física. Se trata de un dolor corporal facilitado por la expansión actual de la biomedicalización de la vida ${ }^{4}$ en la que el malestar no se relaciona directamente con un sentido particular de la experiencia vital y subjetiva sino con el organismo.

"El dolor yo lo tengo siempre, me acuesto con él y no duermo, que esto es muy malo, y me levanto con él" (GF7).

"El día que tienes mucho dolor, que lo aguantas porque no tienes otra, es que no sabes ni hablar, se te va la memoria, no sabes hablar y te irritas más" (GF8).

Por otra parte, dicha vulnerabilidad intercede en el desempeño del rol de género "ser para otros" y en la expresión de la identidad de género, lo que genera culpabilidad y aumenta el dolor.

"Me siento culpable de tener que llevar semanas... como esta semana que no me encuentro nada bien, me encuentro muy jorobada, tener que llevar a por planchar. Y me siento mal, culpable, inútil” (GP).

"Fastidias a los demás. Les cambias la vida" (GFA5).

\section{Yo estigmatizado}

A la pregunta sobre la respuesta social ante del dolor limitante, se responde que resulta ininteligible e invisible 
para el entorno, que conduce a la creación de un estigma social el cual construye a las personas afectadas como diferentes, hasta el punto de hacerles creer que son mentirosas, vagas, resentidas, locas, etc.:

"Vas a un médico, te hacen una radiografía y te dicen: sí que tienes una artrosis pero no responde al dolor que usted dice que tiene. Te manda a otro. Le dices el mismo problema, porque tienes aquél y no le puedes explicar otro, y te das cuenta de que te están mirando, mientras se están riendo" (GP).

Este estigma también está presente en las inspecciones médicas, donde se dirimen derechos sociales de prestaciones económicas por invalidez:

"Incluso el ICAM te cuestiona, que eres una vaga, ¡después de que has dejado tu vida! (...) ¡Es vergonzoso! y me he tenido que justificar delante un médico que me cuestiona como persona" (GF3).

Se abre una brecha profunda entre el conocimiento experto y el conocimiento experiencial silenciado de las afectadas.

\section{Organizaciones de afectadas y empoderamiento del yo}

Cuando se pregunta sobre la aportación de las organizaciones y asociaciones a la vida de las pacientes, surgen diferentes temas. De entrada, se observa que ayudan a diluir el estigma social, a través del diálogo intersubjetivo y del apoyo y reconocimiento mutuo que proporciona:

"Yo tenía miedo. Pensaba que me estaba volviendo loca de verdad, porque es lo que me estaban machacando. Después te das cuenta que a otras personas les pasa lo mismo" (GF6).

Para que esto ocurra, la asociación es construida como rompedora con el encierro y el aislamiento domésticos, propio del modelo de género tradicional:

"Ser de la asociación te permite salir de casa, sino muchas nos quedaríamos en casa" (GFA3).

Además, es frecuente que el dolor se transforme en dolor subjetivo, ya que en el discurso sobre la asociación aparece la dimensión emocional de la experiencia, el placer y el disfrute, así como el displacer (el servicio obligado y continuado a los demás):

"Yo estaba fatal y cuando volví al médico después de tres meses de ir a la asociación, y me dice ¿cómo estás? le digo animicamente muy bien (...) es verdad que me ha ido muy bien, me encuentro mejor" (GFA9).

"Aqui nos olvidamos de todo, del exterior, de todos nuestros males y disfrutamos este momento" (GFA5).

Paradójicamente, la naturaleza del dolor cambia en algún grado y no cambia, pero parece transformarse y diluirse su cronificación. El intercambio entre iguales en la asociación, legitima al sujeto para que escuche su cuerpo y lo piense como parte de una misma, y no como escindido del yo.

"Tu cuerpo te dice que no, y tú también dices no, esto no lo hago, pero antes" (GFA2).

"Antes no podías pero no decías que no, lo hacías" (GFA2).

Dicha integración del cuerpo en el yo conduce a inaugurar e ir construyendo un espacio propio subjetivo, el cual permite transformar la experiencia de dolor corporal en una experiencia psicosocial. Lo que conduce a cuidarse a sí misma un poco más, e ir empoderándose ${ }^{29}$.

"Yo pienso que las mujeres estamos muy estresadas, más que los hombres. Los hombres van a trabajar, llegan a casa y la mayoría se tumban en el sofá. Pues nosotras no, las de nuestra generación, no descansamos ni tenemos tiempo propio" (GFA5)

"Me gustaba todo bien puesto, y limpio, y todo bien hecho (...) ahora una cosa buena que he aprendido con el tiempo, y la asociación, es a decir que no, que no puedo o no quiero, y a cuidarme" (GFA8).

Se observa cómo este proceso de empoderamiento (que consigo viene la posibilidad de decir no y cambiar la vida cotidiana) permite en algún punto, transformar la narración inicial sobre la diferencia sexual biológica como causa del dolor. Ésta queda desplazada por una consciencia incipiente de desigualdad de género que abre posibilidades de agencia y de transformación social mayores. 


\section{DISCUSIÓN}

A pesar de las críticas recibidas, los resultados muestran que la salud y el bienestar siguen estando bajo la tutela hegemónica de la aproximación biopsicomédica, la cual en general restringe y limita las formas de describirla, explicarla e intervenir sobre ella. Esto es especialmente cierto en relación a malestares emergentes, como la Fibromialgia en la que lo bio es un argumento débil, y que surgen en las sociedades occidentalizadas, neoliberales y tecnologizadas.

Cuando el dolor se cronifica sin causa aparente la vida se trunca, pero los sistemas de salud basados en perspectivas biomédicas no ofrecen explicaciones ni tratamientos específicos, razón por la cual el estigma social está servido, y más cuando afecta específicamente a un colectivo psicosocialmente vulnerable. Estudios como el de Bustillo-Guzmán et al $^{29}$ y Campos-Arias y Herazo $^{30}$ muestran resultados similares en problemas relacionados, cuando no se incorporan variables y dimensiones psicosociales en situaciones de riesgo y vulnerabilidad. Las organizaciones de afectadas surgen como producto de este vacío. Los resultados evidencian cierto desplazamiento desde una subjetivación de género normativa hacia el empoderamiento así como una emergente toma de consciencia sobre la desigualdad de género, a partir de la participación en dichas organizaciones. Esto supone cierta transformación de la experiencia del dolor corporal en experiencia psicosocial, la cual ayuda a deshacer el proceso de cronificación del dolor al convertirse en experiencia vital con un sentido específico.

Respecto a los discursos de las pacientes afectadas pueden distinguirse dos aspectos diferentes. El primero es que en estos discursos puede leerse una reproducción de los discursos biopsicomédicos androcéntricos hegemónicos, en la que se adopta una posición de objeto y no de sujeto de experiencia. Este se convierte en un factor que dificulta la recuperación y agudiza el padecer. Sin embargo, el segundo aspecto involucra la emergencia de un discurso donde emerge un nuevo dolor subjetivo, acumulado en sus trayectorias vitales, que expresa una subjetivación normativa de género. Subjetivación que se corresponde con un estilo de vida en el que no hay espacio subjetivo propio, lo que desde el enfoque de género en salud, se explica cómo la presencia de un conflicto interior continuo entre los mandatos sociales de género de servir al otro y las necesidades particulares. Aunque se trata de un conflicto inconsciente, puesto que la identidad de género está regulada por mandatos y normas sociales que el sujeto incorpora como si se tratara de deseos propios, con el fin de obtener reconocimiento social, aunque sea a partir del sometimiento ${ }^{31}$. Trabajo como el de MontesóMurto $^{32}$ ha mostrado la importancia de incorporar un enfoque de género en salud no solo en el tratamiento del dolor crónico y la fibromialgia, sino también en otros problemas de salud como el estrés y la depresión.

El estudio muestra que urge introducir con mayor determinación y profundidad, en la definición y organización del cuidado de la salud, el análisis del impacto de factores psicosociales no saludables, entre ellos los distintos modelos sociales de desigualdad de género, adaptados al contexto. Resultados similares pueden encontrarse en estudios realizados con personal médico en el trabajo con fibromialgia, en el cual declaran la poca preparación que hay para tratar de esta naturaleza ${ }^{33}$. Así como re-pensar la intervención en coherencia con esta perspectiva, para que el abordaje no sea meramente psico-farmacológico y/o basado en la terapia cognitivo-conductual, hegemónica en España, cuyo efecto muchas veces es psico-patologizador, cronificando el dolor. Este cambio supone un gran desafío, que es la articulación efectiva entre las Ciencias Sociales y Humanas y las Ciencias de la Salud en el abordaje de la experiencia de malestar contemporánea. Sin ésta, los malestares emergentes quedan desamparados, siendo responsabilidad de los/as profesionales involucrados reconocerlos y mitigarlos.

\section{DECLARACIÓN SOBRE CONFLICTOS DE INTERESES}

Este artículo pertenece al proyecto "Les veus silenciades en temps "d'igualtat. El dolor des d'una perspectiva de gènere" financiado por el Institut Catala de Dones, organismo público dependiente de la Generalitat de Catalunya. Sin embargo, el proyecto se ha realizado con total autonomía, sin tener conflictos de interés en sus procesos y resultados.

\section{REFERENCIAS BIBLIOGRÁFICAS}

1. Velasco S. Atención biopsicosocial al malestar: intervención en atención primaria de salud. Instituto de la Mujer; 2006. 
2. Velasco S. Sexos, género y salud: teoría y métodos para la práctica clínica y programas de salud. Madrid: Minerva Ediciones; 2009.

3. Pujal i Llombart M, Mora E. Subjectivity, health and gender: an approach to chronified pain through the Psychosocial Gender Diagnostic methodology. Estudios de Psicología: Studies in Psychology. 2014; 35(2):212-38.

4. Barker K. The Fibromyalgia Story. Medial authority and women's worlds of pain. Philadelphia: Temple University Press; 2005.

5. Luz TM. El desafío de la salud en las ciencias sociales: el caso de Brasil. Política y Sociedad. 2011; 48(2):313-27.

6. Arruda-Leal A. La experiencia de subjetividad como condición y efecto de los saberes y las prácticas psicológicas: producción de subjetividad y psicología. Estudios de Psicología. 2001; 32(3):359-74.

7. De Vogli R. Neoliberal globalisation and health in a time of economic crisis. Social Theory and Health. 2011; $9(4): 311-25$.

8. Ramos J. La histeria camaleónica y la seducción de los nuevos diagnósticos. Cuadernos de Salud Mental Aproximaciones contemporáneas a la histeria. 2009; 12(1):189-212.

9. Gómez V, Pérez LA, Feldman L, Bajes N, Vivas E. Riesgos de salud en mujeres con múltiples roles. Revistas de Estudios Sociales. 2000;(6):27-38.

10. Hospital Universitario Vall d'Hebron. XI Jornada de avances en el síndrome de fatiga crónica. Barcelona; 2013.

11. Velasco S, Doriga BL, Garcia MT, Barceló IB, Rodríguez CL. Evaluación de una intervención biopsicosocial para el malestar de las mujeres en atención primaria. Feminismo/s: revista del Instituto de Investigación de Estudios de Género de la Universidad de Alicante. 2007;(10):111-31.

12. Ubago M del C, Ruiz I, Bermejo MJ, Olry A, Plaza J. Características clínicas y psicosociales de personas con fibromialgía. Repercusión del diagnóstico sobre sus actividades. Revista Española de Salud Pública. 2005;(79):683-95.

13. Rubin G. El tráfico de las mujeres: notas sobre la economía política del sexo. Nueva Antropología. 1986; 8 (20): 95-145.

14. Pujal i Llombart M, Amigot Leache P. El binarismo de género como dispositivo de poder social, corporal y subjetivo. Quaderns de Psicologia International Journal of Psychology. 2010; 12(2):131-48.

15. Faro M, Sàez-Francás N, Castro-Marrero J, Aliste L, Fernández de Sevilla T, Alegre J. Diferencias de género en pacientes con síndrome de fatiga crónica. Reumatología Clínica. 2016;12(2):72-7.
16. Alvarado-Moreno JG, Montenegro-Díaz KA, LeónJiménez FE, Díaz-Vélez C. Association between Depression-Anxiety and the Fibromyalgia syndrome in 3 health centers of Lambayeque, Peru, 2011-2012. Revista Médica de Risaralda. 2014;20(2):75-9.

17. Collado Cruz A. Fibromialgia. Madrid: Ministerio de Sanidad, Política Social e Igualdad; 2011.

18. García O. Espectro ansioso-depresivo-somatizador, fibromialgia y síndrome de fatiga crónica. Revista de Medicina General y de Familia. 2007;(93):105-9.

19. Mora E. Patriarcado, capitalismo y clases sociales. En: El género quebrantado Sobre la violencia, la libertad y los derechos de la mujer en el nuevo milenio. Madrid: La Catarata; 2005. p. 143-8.

20. Iñiguez L, Monreal P, Sanz J, del Valle A, Fuste P. Análisis integrado de las percepciones de los ciudadanos, los profesionales y los gestores sobre la atención sanitaria en Catalunya. Forum Qualitative Sozialforschung/Forum: Qualitative Social Research; 2009;(10)2:Art. 34.

21. Goffman E. Asylums essays on the social situation of mental patients and other inmates. Nueva York: Doubleday; 1961.

22. Denzin N, Lincoln Y. Introduction: The discipline and practice of qualitative research. En: Denzin N, Lincoln Y, editores. Handook of Qualitative Research. 3a ed. Londres: SAGE; 2005. p. 1-32.

23. Morgan DL, Spanish MT. Focus groups: A new tool for qualitative research. Qualitative Sociology. 1984;7(3):253-70.

24. Calder B. Focus groups and the nature of qualitative marketing research. Journal of Marketing Research. 1977;(14):353-64.

25. Kohlbacher F. The use of qualitative content analysis in case study research. Forum Qualitative Sozialforschung/ Forum: Qualitative Social Research. 2006;7(1):Art. 21.

26. López Noguero F. El análisis de contenido como método de investigación. XXI Revista de educación. 2002;(4):167-80.

27. Piñuel JL. Epistemología, metodología y técnicas del análisis de contenido. Sociolinguistic Studies. 2002;3(1):1-42.

28. Lagarde M. Para mis socias de la vida. Madrid: Horas y Horas; 2005.

29. Bustillo-Guzmán M, Rojas-Meriño J, Sánchez-Camacho A, Sánchez-Puello L, Montalvo-Prieto A, Rojas-López M. Riesgo psicosocial en el personal de enfermería. Servicio de urgencias en hospital universitario de Cartagena. Duazary. 2015;12(1):32-40.

30. Campo-Arias A, Herazo E. Pobreza, desigualdad y tasa de suicidio en Colombia, 2012. Duazary. 2014;11(2):126-30. 
31. Butler J. Mecanismos psíquicos del poder. Madrid: Editorial Cátedra; 2001.

32. Montesó-Curto P. La construcción de los roles de género y su relación con el estrés crónico y la depresión en las mujeres. Comunitania Revista Internacional de Trabajo Social y Ciencias Sociales. 2014;(8):105-26.
33. Hayes SM, Myhal GC, Thornton JF, Camerlain M, Jamison C, Cytryn KN, et al. Fibromyalgia and the therapeutic relationship: Where uncertainty meets attitude. Pain Research and Management. 2010;15(6):385-91.

Para citar este artículo: Pujal i-Llombart M, Mora-Malo E, Schöngut-Grollmus N. Fibromialgía, desigualdad social y género. Estudio cualitativo sobre transformación del malestar corporal en malestar psicosocial. Duazary. 2017 enero; 14 (1): 70 - 78 